\title{
¿SOLAMENTE O SOLAMENTO?: DESAFÍOS EN EL APRENDIZAJE DE FORMA Y SIGNIFICADO DE -MENTE Y -MIENTO/-MENTO POR HABLANTES DE ELE
}

\author{
Nausica Marcos Miguel \\ Denison University, EE. UU.
}

\author{
Claudia Helena Sánchez Gutiérrez \\ University of California, Davis
}

\section{RESUMEN}

Este estudio se aproxima a las dificultades más frecuentes que enfrentan los estudiantes de español como L2 (EL2) en la adquisición del significado y forma de los sufijos -mente y -mientol-mento. En el Corpus de Aprendices de Español (CAES), se localizan palabras derivadas con estos sufijos, y se codifican errores formales y semánticos que indiquen si los aprendices utilizan las palabras de memoria o las descomponen. Los errores semánticos son limitados y se ajustan a palabras específicas. Tampoco destacan errores sintácticos en la asignación de la categoría gramatical de la palabra. Los errores ortográficos y morfológicos son los más frecuentes. Conforme al modelo de Jiang (2000), los aprendices parecen unir primero la forma de toda la palabra con el significado en la L1. Tras esta etapa, descomponen la palabra, lo que se refleja en sus errores ortográficos y morfológicos. Estos errores indicarían que el sufijo se guarda como un elemento de la L2 en el lexicón. Con los ejemplos analizados, se ofrece una serie de palabras prototípicas para que los aprendices de EL2 reflexionen sobre estos sufijos.

Palabras Clave: ELE, morfología, sufijos, análisis de corpus, CAES.

\section{CHALLENGES IN LEARNING THE FORM AND MEANING \\ OF THE SUFFIXES -MENTE AND -MIENTO/-MENTO BY SPANISH L2 LEARNERS:}

A CORPUS-BASED STUDY

\section{Abstract}

This corpus-based study addresses the most frequent difficulties that Spanish L2 learners show in the acquisition of meaning and of the frequent suffixes -mente and -miento/-mento. In the Corpus de Aprendices de Español (CAES), derived words with these suffixes were searched. Meaning-based and formal errors were coded to show whether learners were using whole word memorization or whether they were decomposing words. Meaning-based errors were limited and related to specific words. Syntactic errors were also scarce. Orthographic and morphological errors were the most frequent. According to Jiang's (2000) model, learners seem to make connections between the L2 word form and the L1 meaning. After this phase, learners start decomposing the word, which is reflected in their orthographic and morphological errors. These errors point out that the suffix is stored in the L2 lexicon. Based on the examples analyzed, some prototypical words are recommended for awareness raising activities so that Spanish L2 learners recognize suffix' characteristics and their learning challenges.

KEYwORds: Spanish L2, morphology, suffixes, corpus-based analysis, CAES.

DOI: https://doi.org/10.25145/j.refiull.2020.40.08

Revista de Filología, 40; enero 2020, pp. 147-165; ISSN: e-2530-8548 


\section{INTRODUCCIÓN}

La enseñanza/aprendizaje de la morfología derivativa en las clases de espanool lengua extranjera (ELE) está ganando tracción en este momento (Díaz Hormigo, 2011; Marcos Miguel, 2016, 2017, 2018; Martínez Vázquez, 2016; Morin, 2003, 2006; Robles García y Sánchez Gutiérrez, 2016; Sánchez Gutiérrez, 2013; Sánchez Gutiérrez, Marcos Miguel y Robles García, 2020; Serrano-Dolader, s.f.). Prueba de ello son obras como el reciente libro de Serrano-Dolader (2019), donde el autor revisa diferentes afijos del español, reflexionando sobre sus usos y formas y proponiendo actividades para introducirlos en el aula. Este hincapié en la morfología derivativa es bienvenido ya que la mayoría de materiales de enseñanza de español, tanto de L1 como de L2, apenas le dedican espacio (Montero Curiel, 2009).

Sin embargo, si bien se están multiplicando las propuestas didácticas en este campo, aún se sabe poco sobre las dificultades específicas que presentan los afijos del español para aprendices de diferentes L1. Por ejemplo, cabe preguntarse cuál es el desarrollo formal y semántico de los sufijos en hablantes con lenguas de distinta tipología. En efecto, es necesario averiguar si las dificultades de los estudiantes se deben principalmente a cuestiones formales (e. g., cambiar el adjetivo a su forma femenina antes de crear un adverbio terminado en -mente) o semánticas (e.g., atribuir un significado a un sufijo, incluso cuando no hay sufijos correspondientes en la L1). Propuestas de adquisición del léxico como las de Jiang (2000) en las que primero se asocia una palabra nueva con la L1 y poco a poco se va desarrollando una nueva entrada en el lexicón de la L2 pueden ayudarnos a entender el proceso de adquisición de palabras derivadas.

Estas investigaciones sobre morfología tienen influencia directa en la instrucción de L2 si seguimos las recomendaciones del modelo "four strands» (cuatro aspectos) (Nation, 2007). Este modelo se basa en las investigaciones de adquisición de L2 que han mostrado que tanto la práctica del significado como de la forma son fundamentales para aprender una L2. Con este modelo las clases de lengua deben dedicarse a tareas que incluyan:

1) input, oral y escrito, para que los estudiantes trabajen el significado (meaningfocused input),

2) producción, oral y escrita, donde los estudiantes se comuniquen (meaning-focused output),

3) tiempo para enfocarse en las formas lingüísticas (language-focused learning), y

4) práctica de fluidez (fluency development).

La instrucción de la morfología derivativa explícita se sitúa en el tercer aspecto. Sin embargo, no contamos con información sobre sufijos específicos que transmitirles a los docentes de L2. La mayoría de los estudios sobre conciencia morfológica en segundas lenguas han utilizado tareas controladas en las que se incluían palabras con afijos muy diferentes entre sí. Es decir, en una misma prueba podían aparecer palabras terminadas en -eza, un sufijo muy poco frecuente en español y sin una clara asociación semántica o formal con sufijos del inglés, por ejemplo, y pala- 
bras terminadas en -ción, un sufijo muy frecuente cuya forma es similar en francés o inglés (i. e., -tion). El resultado final de dichas pruebas, al agregar los datos de ambos sufijos, no revela si estos siguen patrones similares o no, lo cual no permite hacer un diagnóstico detallado de las dificultades específicas que presentan para el aprendiz de ELE en diferentes momentos de su desarrollo lingüístico. Y mientras no sepamos por qué y cuándo surgen dichas dificultades, será imposible ofrecer propuestas didácticas que respondan a las necesidades reales de los aprendices. Debido a que los datos disponibles sobre conciencia morfológica en ELE provienen principalmente de pruebas muy controladas (Sánchez Gutiérrez y Hernández Muñoz, 2018), falta aún información sobre el uso real, en contexto de producción escrita u oral, de morfemas derivativos. En efecto, son muy pocos los trabajos que han analizado la utilización de afijos y errores de formación de palabras en aprendices de ELE (Fernández, 1996; Whitley, 2004; Zyzik y Azevedo, 2009) y ya hay autores, en inglés o en alemán, que han defendido la necesidad de utilizar datos de corpus para analizar el uso productivo de morfemas en aprendices de segundas lenguas (Callies, 2016; Lüdeling, Hirschmann y Shadrova, 2017).

En este trabajo pretendemos solventar estos problemas, limitando nuestro análisis a dos sufijos de alta frecuencia en español: -mente y -miento/-mento y analizando su utilización en palabras reales (e.g., solamente, apartamento), inventadas (e.g., ${ }^{*}$ entiendamiento, ${ }^{*}$ amicalmente) y formalmente erróneas (e.g., ${ }^{*}$ solamento, ${ }^{*}$ appartemente) en un corpus de textos escritos por estudiantes de ELE de diversos niveles y L1 (CAES, Corpus de Aprendices de Español, Rojo y Palacios, 2016). El objetivo es averiguar qué aspectos del uso de estos sufijos relacionados con el modelo de Jiang (2000) presentan dificultades para los aprendices de ELE y proponer maneras concretas de atajar dichas dificultades.

\subsection{Modelos Del DeSARRollo MORFolóGico}

La conciencia morfológica en los niños que adquieren una primera lengua (L1) se desarrolla, según Tyler y Nagy (1989), en tres etapas. La primera se centra en el dominio de los conocimientos relacionales, que consisten en reconocer que las palabras complejas tienen una estructura interna y que varias palabras pueden compartir un mismo morfema. A este tipo de conocimientos se asocian habilidades como las de reconocer la raíz común en palabras de una familia léxica, como aceptar, aceptable y aceptación, por ejemplo. Posteriormente se desarrollan los conocimientos sintácticos, que permiten establecer la categoría gramatical que aportan los afijos derivativos a las bases a las que se aplican. Este tipo de conocimiento se relaciona con la capacidad para comprender que -ción se utiliza para formar sustantivos mientras que -ble forma adjetivos. Finalmente, se adquieren los conocimientos combinatorios que conllevan una toma de conciencia de las restricciones morfológicas que subyacen a la estructura de las palabras y permiten descubrir que -ble solo se puede aplicar a bases verbales, mientras que -ista se aplica a bases nominales.

Nos encontramos con que tanto los aprendices de una L1 como de una L2 desarrollan su competencia morfológica mediada por la escritura (ver Kuo y Ander- 
son, 2006). Por ejemplo, para reconocer y producir en la escritura un morfema, un estudiante necesita tanto conocimientos ortográficos y fonológicos como semánticos.

Si bien el modelo de Tyler y Nagy (1989) podría servir de base para establecer hipótesis sobre cómo desarrollan su conocimiento morfológico los estudiantes de L2, presenta dos limitaciones sustanciales: (1) se centra en aspectos receptivos del conocimiento morfológico, dejando de lado cuestiones de producción y sin considerar el conocimiento ortográfico o fonológico de la forma del sufijo, y (2) no tiene en cuenta el componente semántico de los morfemas. Con respecto al punto (1), este modelo no permite plantear hipótesis sobre el momento en el que los aprendices activamente aplican el sufijo -mente a la forma femenina de un adjetivo o en el que utilizan correctamente la vocal temática correspondiente al tipo de verbo al juntar -miento a la base verbal. En relación con el punto (2), cada una de las tres etapas propuestas se centra en cuestiones formales, sintácticas o categoriales, pero no se menciona ninguna etapa en la que los aprendices descubran el significado de los afijos de una lengua. Esto es relevante, sobre todo cuando tenemos en cuenta el modelo de adquisición léxica en L2 propuesto por Jiang (2000), donde el componente semántico es central y está muy relacionado con la L1. Concretamente, el autor propone que el desarrollo del conocimiento léxico sigue tres etapas:

1. En un primer momento, los aprendices descubren la forma de una palabra nueva en la L2 e inmediatamente la conectan con la entrada léxica de la palabra correspondiente en la L1. Esta etapa se caracteriza por la falta de una representación semántica y/o sintáctica propia de la L2.

2. En una segunda etapa, las características semánticas y sintácticas de la L1 se integran dentro de la entrada léxica de la palabra en la L2, permitiendo una mayor automatización en la recuperación de información del lexicón del estudiante. Sin embargo, en este punto aún no se incluye ninguna información propia de la L2, salvo la forma de la palabra.

3. En la última etapa, se integra información sobre la palabra que es específica de la L2, de manera que la entrada léxica de la palabra pasa de incluir solamente información de la palabra correspondiente en la L1 a incluir las formas flexivas de la palabra, u otras características que no podrían extraerse de la $\mathrm{L} 1$, como el reconocimiento de su estructura interna.

Jiang (2000) también explica que es habitual que se den fenómenos de fosilización léxica, de forma que los aprendices solo obtienen una representación parcial de las palabras de la L2, basada en la L1. Esto significa que no se llega a la tercera etapa del modelo en muchas de las palabras en la L2. En el contexto de la morfología derivativa, esto podría significar que, si el reconocimiento de la estructura morfológica de la palabra solo viene en la última etapa, pocos aprendices llegan a dominar bien las reglas de formación de palabras, a menos que su L1 presente patrones de formación de palabras ( $y$ afijos) similares que permitan el desarrollo de este conocimiento incluso en la primera etapa del desarrollo léxico.

No podemos olvidar que, en procesos psicolingüísticos, la adquisición de la morfología derivativa es diferente de la flexiva. Mientras que para la flexiva pode- 
mos esperar una mayor capacidad de descomposición de la estructura de la palabra, esto no resulta siempre así en la derivativa (ej.. Clahsen y Felser, 2017). Sin embargo, este análisis, al trabajar con producción y además escrita, se acerca más a los estudios de orden de adquisición de morfemas flexivos que a estudios de corte psicolingüístico. Consideramos este enfoque necesario debido al valor de los corpus y las metodologías de lingüística de corpus para explorar conocimientos morfológicos en la L2 (ej., Lozano y Díaz-Negrillo, 2019; Murakami y Alexopoulou, 2017) y a la importancia de los conocimientos explícitos en el aprendizaje de lengua (ej., Kuo y Anderson, 2006; Nation, 2007).

\subsection{Hipótesis PREVIAS}

En conclusión, si el conocimiento morfológico está principalmente mediado por la L1, cuanto más tipológicamente diferentes sean la L1 y la L2, más dificultades habrá para desarrollar un conocimiento morfológico profundo y productivo. Esto se verá reflejado en la cantidad de palabras con variación formal respecto a las formas aceptadas en la L2 (e.g., normalmente, *normalaamente, *normalemente, *normalmemte, etc.) o, incluso, inventadas a partir de reglas de formación de palabras (e.g., *ontemimiento en lugar de contenido), ya que estas son las que pueden revelar si los estudiantes tienen un conocimiento productivo de las reglas de formación de palabras y/o de un sufijo dado. Además, es necesario aproximarse a distintos niveles de competencia en la L2. Por ejemplo, respecto a -mente, basándose en una prueba de producción, Baralo (1994) considera que en hablantes avanzados no hay diferencias entre L1 en su producción y, salvo por algunos casos con adjetivos deverbales, producen formas similares a los nativos. Aunque podemos esperar un resultado final equivalente al de los nativos, todavía nos queda explorar el desarrollo en niveles elementales e intermedios.

La tabla 1 resume las similitudes formales, semánticas y de reglas de formación de palabras entre -mente y los sufijos correspondientes (si los hay) en las diferentes L1 estudiadas aquí. Según las características de las diferentes lenguas, sería esperable que los aprendices de chino sean los que menos descompongan las palabras en su lexicón y de ahí que tampoco creen nuevas, aunque utilicen de manera productiva palabras reales con este sufijo que hayan aprendido como una unidad léxica no descomponible. Los aprendices de L1 árabe, en cambio, al tener un sufijo correspondiente a -mente en su L1, podrían aprovechar esta similitud semántica (pero no formal) para descomponer desde el principio la palabra e incluso crear más adverbios nuevos en -mente. En cuanto a los hablantes de L1 inglés y francés, ambos tienen acceso a sufijos con el mismo valor en sus respectivas lenguas, aunque el sufijo correspondiente en inglés (i. e., $-l y$ ) es formalmente distinto del español mientras que el sufijo francés (i. e., -ment) es un cognado gráfico del español. Esto podría conllevar una adopción más temprana del sufijo -mente en los estudiantes francófonos que en los aprendices anglófonos. Un punto interesante aquí es que el francés es la única lengua cuyo sufijo correspondiente no solo es similar semántica y formalmente, sino que además sigue una misma regla de formación de palabras: el 
sufijo solo puede aplicarse a la forma femenina de los adjetivos. Aunque el sufijo de femenino en francés no sea tan saliente como en español, ya que es reconocible solo a nivel gráfico, los hablantes de francés podrían presentar menos dificultades formales a la hora de aplicar esta regla en la formación de adverbios nuevos en español.

\begin{tabular}{|c|c|c|c|}
\hline \multicolumn{4}{|c|}{$\begin{array}{l}\text { TABLA 1. SIMILITUD FORMAL, SEMÁNTICA, COMBINATORIA Y DE FRECUENCIA } \\
\text { ENTRE EL SUFIJO -MENTEY LOS EQUIVALENTES (SI LOS HAY) EN LAS DIFERENTES L1 }\end{array}$} \\
\hline & Similitud FORMAL & Similitud SEMÁNTICA & Similitud COMBINATORIA \\
\hline Árabe & No & Sí & No \\
\hline Chino & No & No & No \\
\hline Francés & Sí & Sí & Sí \\
\hline Inglés & No & Sí & Sí \\
\hline
\end{tabular}

La tabla 2 resume las similitudes formales, semánticas y de reglas de formación de palabras entre -miento y los sufijos correspondientes (si los hay), en las diferentes L1 estudiadas aquí. Las hipótesis sobre qué estudiantes encontrarán más dificultades según su L1 son similares a las que propusimos en el caso de -miento: los hablantes de L1 chino probablemente recurran poco a -miento para descomponer $\mathrm{y}$ formar palabras nuevas $\mathrm{y}$, por ello, presenten pocos errores de formación de palabras; los aprendices de L1 árabe podrán aprovechar que existen morfemas nominalizadores en su L1, aunque esta ventaja será mínima, dadas las diferencias formales y de formación de palabras entre ambas lenguas; los aprendices de inglés y francés deberían presentar el mayor número de descomposiciones y formas léxicas nuevas en -miento, ya que ambas lenguas tienen un sufijo correspondiente que es un cognado. Sin embargo, esta ventaja se limita a cuestiones formales de ortografía, sintácticas y semánticas, pero pueden surgir dificultades en los aspectos más específicos de la formación de palabras con -miento en español, a saber, que la vocal temática varía según el tipo de verbo al que se aplica el sufijo. No obstante, tanto en inglés como en francés se añade el sufijo cognado a una base verbal.

TABLA 2. SIMILITUD FORMAL, SEMÁNTICA, COMBINATORIA Y DE FRECUENCIA ENTRE EL SUFIJO -MIENTO Y LOS EQUIVALENTES (SI LOS HAY) EN LAS DIFERENTES L1

\begin{tabular}{cccc}
\hline & Similitud Formal & Similitud SEmÁntica & Similitud COMbinatoria \\
\hline Árabe & No & Sí & No \\
\hline Chino & No & No & No \\
\hline Francés & Sí & Sí & No \\
\hline Inglés & Sí & Sí & No \\
\hline
\end{tabular}

Independientemente de la L1, -mente y -miento presentan una dificultad adicional debido a su parecido formal. Es decir, si el reconocimiento de un morfema se basa inicialmente en sus características gráficas, es posible que algunos aprendices empiecen confundiendo ambos sufijos, hasta que consigan asociarles significados distintos y aprender sus características sintácticas propias. Situamos este conocimiento 
formal referido a la ortografía como una primera aproximación al conocimiento del sufijo. En los modelos actuales de conciencia morfológica no es posible separar conocimientos morfológicos de la escritura de los morfemas (ver Fejzo, 2016).

\section{PREGUNTAS DE INVESTIGACIÓN}

1. Conforme avanza el nivel de competencia de español en hablantes de ELE:

a. ¿Cómo se desarrolla el conocimiento de la forma (ortográfica, morfológica y sintáctica) de las palabras con -mente y -miento?

b. ¿Cómo se desarrolla el conocimiento del significado de palabras con los sufijos -mente y -miento?

2. ¿Qué sufijo, -mente o -miento, presenta más dificultades para los estudiantes?

Es decir, ¿dónde deberían insistir más los profesores? ¿Qué palabras pueden usar como ejemplos prototípicos?

\section{METODOLOGÍA}

Este estudio utilizó como fuente de datos el corpus CAES, al que se puede acceder a través de una interfaz disponible online. La tabla 3 refleja el número de casos de los que consta el corpus en los niveles A1-A2 y B1-B2.

\begin{tabular}{ccc}
\hline & TABLA 3. NÚMERO DE CASOS EN CAES \\
\hline & A1-A2 & B1-B2 \\
\hline Árabe & 103374 & 50706 \\
\hline Chino & 33953 & 19210 \\
\hline Francés & 25658 & 28499 \\
\hline Inglés & 60523 & 34583 \\
\hline
\end{tabular}

En la interfaz de CAES se buscaron palabras acabadas en -mente y -miento/mento por nivel (A1, A2, B1 y B2) y L1 (árabe, chino, francés e inglés). Para localizar los ejemplos se escribió un asterisco antes del sufijo. Se eliminaron todos los ejemplos en los que la combinación de las letras -mente y -miento no producía sufijos; ej., la palabra mente. Todos los ejemplos se copiaron en una hoja de Excel agrupándolos en dos niveles: elemental (A1-A2) e intermedio (B1-B2).

A continuación, se codificaron las palabras en función de sus errores: errores semánticos y errores formales (ortográficos, morfológicos y sintácticos). Para los errores semánticos, se observaron dos tipos de errores:

1. el significado de la palabra no funcionaba con la frase (ej., sinceramente, como despedida de una carta);

2. no se usaba una raíz con significado apropiado (ej., * sudamente, como repentinamente, basado en el inglés suddenly). 
Dentro de los errores formales, la categoría «ortográficos» se refiere a los errores en el sufijo (ej., - ${ }^{*}$ memte en vez de -mente). Los morfológicos se dividieron en dos tipos:

1. errores que atañían a la base. Podían ser motivados por un sufijo previo incorrecto

(ej., " fisicalmente en vez de físicamente) o una base incorrecta (ej., * ${ }^{*}$ atentivamente en vez de atentamente);

2. se codificaron como una categoría distinta aquellos que se referían a la vocal temática ya que estos resultaron bastante frecuentes y sistemáticos (ej., especialmente, *especialamente, ${ }^{*}$ especialemente).

Los errores sintácticos también se dividieron en dos tipos:

1. la palabra no funcionaba como un adverbio (en el caso de -mente) o un sustantivo (en el caso de -miento) (ej., "Cuando estoy comparando esta *vacacione y vacaciones de *el pasado, yo pienso que estas vacaciones son *igualamente», igualmente en vez del adjetivo igual,

2. la palabra no estaba colocada en una posición aceptable en español (ej., «mi punto de vista, sobre el *bien estar, ha *totalmente cambiado" en vez de "ha cambiado totalmente»).

Con la herramienta V_words 2.02 (Meara y Miralpeix, 2016), que se puede utilizar con cualquier lengua y se encuentra accesible online, se calcularon por L1 y por nivel (A1-A2; B1-B2) las variaciones de cada palabra. Por ejemplo, normalmente, *normalaamente, *normalemente ${ }^{*}$ normalmemte se contarían como cuatro variaciones de la palabra normalmente. También se incluyeron el número de casos de cada palabra. Es decir, se sumaron los casos de cada palabra con variación. Por ejemplo, si normalmente tenía dos casos, *normalaamente uno, *normalemente tres y *normalmemte dos, el resultado final sería ocho casos, es decir: $2+1+3+2=8$. La información de V_words se copió manualmente en la hoja de Excel de tal manera que se indicaron las variaciones formales y los casos por L1 y nivel.

\section{RESULTADOS}

\section{1. - MENTE (ERRORES SEMÁNTICOS; ERRORES FORMALES: ORTOGRÁFICOS, MORFO- LÓGICOS Y SINTÁCTICOS)}

En la figura 1 se ve que en los textos de inglés y árabe como L1 hay prácticamente el mismo número de tipos en los dos niveles (elemental e intermedio) mientras que en chino y francés hay un incremento de tipos paralelo al aumento en competencia lingüística de los estudiantes. Recordemos que, salvo por francés L1, todas las lenguas tienen más casos en A1-A2 que en B1-B2 (ver tabla 3), lo que quizás haya evitado un mayor incremento en el nivel intermedio. 


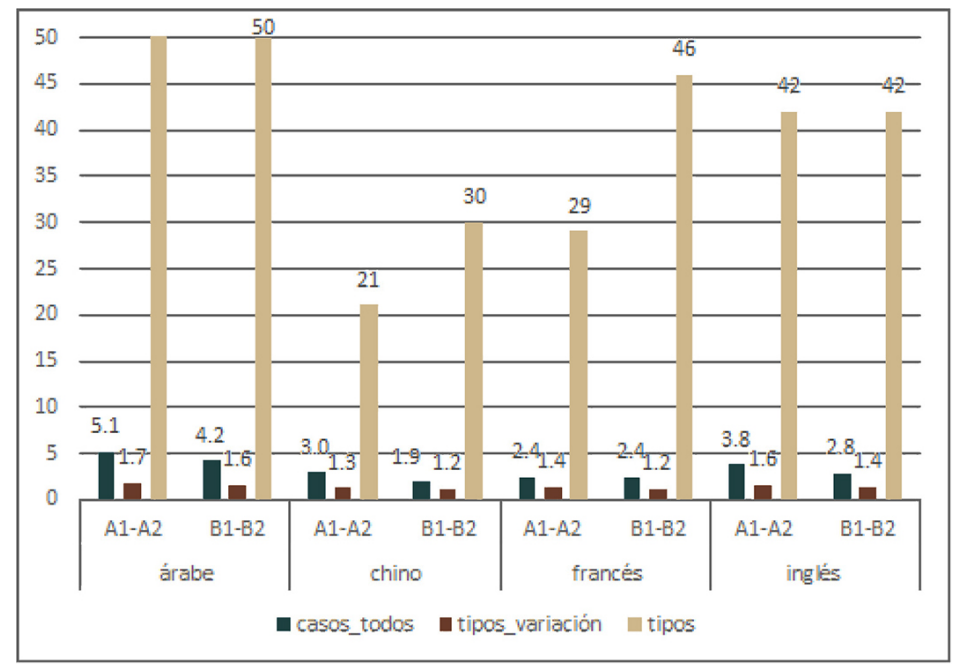

Figura 1. Medias de casos y variación acompañadas de la suma de tipos de palabras con -mente.

Debido al bajo número de errores formales y semánticos, las medias de los errores se recogen en la tabla 4 con sus desviaciones estándares y no en el gráfico anterior. Para los errores formales, las medias se refieren a los errores en cada variación de palabra salvo para los errores sintácticos, que se refieren al total de casos. Este criterio se aplicó también a los errores semánticos. Es decir, la palabra últimamente podía aparecer usada con un significado incorrecto una vez, pero no en todos sus casos. Sin embargo, la palabra *realamente siempre tendría un error formal, independientemente del número de casos. La desviación estándar es bastante elevada, lo que indica que hay mucha variación por palabra.

\begin{tabular}{|c|c|c|c|c|c|c|c|c|c|c|c|c|c|c|c|c|}
\hline \multirow[b]{4}{*}{ error } & \multicolumn{16}{|c|}{$\begin{array}{l}\text { ABLA 4. MEDIAS Y DESVIACIONES ESTÁNDARES DE ERRORES FORMALES } \\
\text { (ORTOGRÁFICOS, MORFOLÓGICOS Y SINTÁCTICOS) Y SEMÁNTICOS } \\
\text { CON PALABRAS CON -MENTE }\end{array}$} \\
\hline & \multicolumn{4}{|c|}{ ÁrABE } & \multicolumn{4}{|c|}{ Chino } & \multicolumn{4}{|c|}{ FRANCÉS } & \multicolumn{4}{|c|}{ INGLÉS } \\
\hline & \multicolumn{2}{|c|}{$\mathrm{A} 1-\mathrm{A} 2$} & \multicolumn{2}{|c|}{ B1-B2 } & \multicolumn{2}{|c|}{ A1-A2 } & \multicolumn{2}{|c|}{ B1-B2 } & \multicolumn{2}{|c|}{ A1-A2 } & \multicolumn{2}{|c|}{ B1-B2 } & \multicolumn{2}{|c|}{ A1-A2 } & \multicolumn{2}{|c|}{ B1-B2 } \\
\hline & M & $\mathrm{DE}$ & M & $\mathrm{DE}$ & M & $\mathrm{DE}$ & M & $\mathrm{DE}$ & M & $\mathrm{DE}$ & $\mathrm{M}$ & $\mathrm{DE}$ & $\mathrm{M}$ & $\mathrm{DE}$ & M & $\mathrm{DE}$ \\
\hline ortográfico & .35 & .71 & .18 & .56 & .33 & .48 & .03 & .18 & .28 & .53 & .09 & .29 & .21 & .81 & .12 & .39 \\
\hline vocal & .29 & .73 & .24 & .63 & .05 & .22 & .00 & .00 & .17 & .47 & .07 & .25 & .48 & .80 & .17 & .49 \\
\hline base & .14 & .35 & .16 & .42 & .10 & .30 & .20 & .48 & .07 & .26 & .11 & .32 & .31 & .60 & .14 & .42 \\
\hline sint. cat. & .02 & .01 & .02 & .14 & .14 & .36 & .03 & .18 & .00 & .00 & .00 & .00 & .14 & .35 & .00 & .00 \\
\hline sint. pos. & .00 & .00 & .02 & .14 & .05 & .22 & .00 & .00 & .00 & .00 & .00 & .00 & .00 & .00 & .05 & .22 \\
\hline sem. base & .04 & .19 & .00 & .00 & .00 & .00 & .00 & .00 & .00 & .00 & .00 & .00 & .00 & .00 & .05 & .31 \\
\hline sem. frase & .14 & .60 & .18 & .77 & .14 & .36 & .20 & .48 & .00 & .00 & .02 & .15 & .31 & .86 & .31 & 1.14 \\
\hline
\end{tabular}

sint.=sintáctico; cat.=categoría; pos.=posición; sem.=semántico 
La tabla 4 indica que apenas hay errores semánticos en la base ni formales por la posición de la palabra. Los hablantes de francés son los que cometen menos errores semánticos y sintácticos de todos los aprendices, seguidos por los anglohablantes. Por otro lado, los errores formales relacionados con la ortografía y la vocal temática son los más frecuentes en todas las lenguas. Sin embargo, los hablantes de chino apenas cometen errores con la vocal temática.

\section{2. - MENTE: TIPOS Y SIMILITUDES ENTRE LENGUAS}

Más de un cuarto de las palabras en -mente son usadas en al menos tres lenguas en los niveles A1-A2 y más de la mitad en dos lenguas o más. Dentro de cada lengua hay variación a la hora de escribir la forma de la palabra, que va desde una a dos formas o incluso de dos a siete formas. La tabla 5 recopila las palabras comunes a más de tres lenguas en nivel elemental con más de dos formas en al menos una lengua. Que hubiera una sola forma, o incluso más, no implicaba que la palabra estuviera escrita según es recogida en el $D R A E$. Por ejemplo, para la palabra impacientemente se recoge la forma correcta en árabe, A1-A2, mientras que, para árabe, B1-B2, solo se encuentra la forma ${ }^{*}$ impaciamente. Aunque se indica que la palabra se usa también en B1-B2, esta incluye tanto un error en la base como en la vocal temática.

\begin{tabular}{|c|c|c|c|}
\hline \multicolumn{4}{|c|}{$\begin{array}{l}\text { TABLA 5. PALABRAS COMUNES EN MÁS DE TRES LENGUAS CON -MENTE (A1-A2) } \\
\text { CON MÁS DE UNA VARIACIÓN DE SU FORMA EN AL MENOS UNA DE LAS LENGUAS }\end{array}$} \\
\hline Nivel & Palabra & VARIACIÓN EN CADA LENGUA & LENGUAS (3 O MÁS) \\
\hline \multirow{17}{*}{ 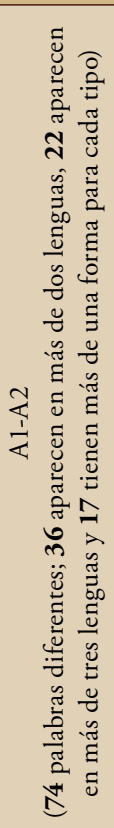 } & absolutamente & 1 a 2 & 4 \\
\hline & actualmente & 1 a 4 & 4 \\
\hline & aproximadamente & 1 a 4 & 4 \\
\hline & especialmente & 2 a 7 & 4 \\
\hline & exactamente & 1 a 4 & 3 \\
\hline & fácilmente & 1 a 2 & 3 \\
\hline & físicamente & 2 a 6 & 3 \\
\hline & finalmente & 2 & 3 \\
\hline & frecuentemente & 1 a 4 & 3 \\
\hline & generalmente & 2 a 5 & 4 \\
\hline & normalmente & 1 a 5 & 4 \\
\hline & perfectamente & 1 a 3 & 4 \\
\hline & realmente & 1 a 2 & 3 \\
\hline & rápidamente & 2 a 3 & 4 \\
\hline & solamente & 1 a 6 & 4 \\
\hline & totalmente & 1 a 2 & 3 \\
\hline & últimamente & 1 a 2 & 4 \\
\hline
\end{tabular}




\begin{tabular}{|c|c|c|c|c|}
\hline \multicolumn{5}{|c|}{$\begin{array}{l}\text { TABLA 6. PALABRAS COMUNES CON -MENTE EN MÁS DE TRES LENGUAS (B1-B2) } \\
\text { CON MÁS DE UNA VARIACIÓN DE SU FORMA EN AL MENOS UNA DE LAS LENGUAS }\end{array}$} \\
\hline Nivel & Palabra & $\begin{array}{l}\text { VARIACIÓN EN CADA } \\
\text { LENGUA }\end{array}$ & $\begin{array}{l}\text { LENGUAS } \\
\text { (3 O MÁS) }\end{array}$ & $\begin{array}{l}\text { EJEMPLOS CON ERRORES } \\
\text { FORMALES }\end{array}$ \\
\hline \multirow{14}{*}{ 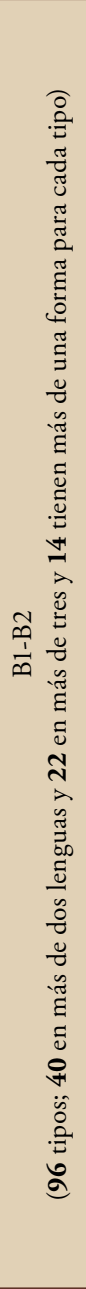 } & actualmente & 1 a 2 & 3 & $\begin{array}{l}\text { actualamente } \\
\text { actualemente }\end{array}$ \\
\hline & aproximadamente & 1 a 3 & 3 & $\begin{array}{l}\text { aproxivamente } \\
\text { aproximente }\end{array}$ \\
\hline & atentamente & 1 a 4 & 4 & $\begin{array}{l}\text { atentemente } \\
\text { atentivamente }\end{array}$ \\
\hline & desafortunadamente & 1 a 3 & 4 & $\begin{array}{l}\text { desafortunamente } \\
\text { desafordunamente } \\
\text { desafortunadamene } \\
\text { desafortunatamente }\end{array}$ \\
\hline & desgraciadamente & 2 & 3 & $\begin{array}{l}\text { desgradaciamento } \\
\text { desgratiatamente }\end{array}$ \\
\hline & directamente & 1 a 2 & 3 & --- \\
\hline & especialmente & 1 a 6 & 4 & $\begin{array}{l}\text { espacialemente } \\
\text { especilemente } \\
\text { spescialment }\end{array}$ \\
\hline & fácilmente & 1 a 5 & 4 & $\begin{array}{l}\text { facialmente } \\
\text { facilamente } \\
\text { facilemente }\end{array}$ \\
\hline & finalmente & 1 a 3 & 4 & $\begin{array}{l}\text { finalmente } \\
\text { finalemente } \\
\text { finalmently }\end{array}$ \\
\hline & realmente & 1 a 2 & 3 & $\begin{array}{l}\text { realememte } \\
\text { realament } \\
\text { realment }\end{array}$ \\
\hline & rápidamente & 1 a 3 & 4 & rápidamente \\
\hline & solamente & 1 a 5 & 4 & $\begin{array}{l}\text { solo mente } \\
\text { solamnete } \\
\text { solomente } \\
\text { solomentse }\end{array}$ \\
\hline & totalmente & 1 a 2 & 4 & todalmente \\
\hline & últimamente & 1 a 3 & 3 & ultamente \\
\hline
\end{tabular}

$\mathrm{Al}$ agrupar todas las palabras acabadas en -mente sin distinción de L1, sí que hay un aumento claro en B1-B2: de 74 se pasa a 96. No obstante, solo hay cuatro palabras que se empiezan a usar en más de dos lenguas (de 36 a 40) y se mantiene el mismo número de tipos en más de tres lenguas: 22. Al comparar las dos tablas se ve que disminuye el número de variación en cada lengua, es decir, cada vez hay más tipos que solo presentan una forma. La tabla 6 refleja las palabras comunes en más de tres lenguas en nivel intermedio. En negrita se enfatizan las palabras compartidas en A1-A2 en más de tres lenguas. En estas palabras en negrita se incluyen también las que no tenían variabilidad en su forma, es decir, que solo había un modo 
de escribirlas; por ejemplo, atentamente, y por tanto no se recogen en la tabla 5. A la tabla 6 de nivel intermedio se le ha ańadido una columna más con los ejemplos relacionados con la forma que se han encontrado en CAES.

\section{3. -MIENTO (ERRORES SEMÁNTICOS Y FORMALES: ORTOGRÁFICOS, MORFOLÓGICOS Y SINTÁCTICOS)}

Para las palabras con -miento se realiza a continuación el mismo análisis que para las palabras con -mente. La figura 2 representa el número total de tipos de palabras derivadas con el sufijo -miento. Vemos que el número total va de cinco (francés, A1-A2) a 20 (árabe, B1-B2). Este valor aparece etiquetado con la palabra «tipos» en el gráfico. Hay un incremento en el número total de tipos al igual que de casos por nivel. El árabe destaca con el mayor número de ejemplos y el chino con el menor. En total, hay 42 tipos para analizar.

En la figura 2, la etiqueta "tipos_variación» indica la media de formas de cada tipo. Aparecen de 1 caso (A1-A2, chino) a 2.1 casos (A1-A2, árabe). En esta categoría se incluyen formas existentes y no existentes en español y se incluyen formas en singular y plural; ej., apartamento, apartamiento (recogido en DRAE), apartamentos $\mathrm{y}^{*}$ apartamente.

La media de «casos_todos» se calculó sumando los casos en cada variación de la palabra. La media va desde 1.5 casos (A1-A2, chino) a 3.9 casos (B1-B2, árabe). En general, aumentan los casos por nivel, al igual que los tipos. La variación de los tipos se mantiene en ambos niveles.

Los errores formales se reflejan en la tabla 7. Los errores ortográficos en el sufijo tratan variaciones tales como usar el alomorfo no habitual; ej., *departamiento en vez de departamento, o cambiar la $n$ por una $m$, *miemto. No llegan a uno por tipo, de 0 (inglés A1-2) a 0.77 (A1-A2, árabe). Los errores de la vocal temática indican la utilización de otra vocal tal como la $e$ o la $i$ en vez de la $a$; ej., ${ }^{*}$ pensimientos en vez de pensamientos, de 0 (chino, A1-A2, B1-B2; francés, B1-B2) a 0.54 (A1-A2, árabe). Los errores en la base, seis en total, consisten en usar otra base que no exista en español; ej., *tournamento en vez de competición, o una base errónea; ej., *compartamiento en vez de comportamiento.

Los errores sintácticos son muy limitados. Apenas se usan palabras derivadas en -miento con otra función que no sea la de sustantivo; ej., el conejo y ella fueron las personas aislamiento de la isla en vez de "aisladas» (inglés, B1-B2). Sin embargo, no se debe olvidar que hemos encontrado dos casos, ${ }^{*}$ solamento y *desgradaciamento, que se han clasificado como errores ortográficos de -mente. Estos mismos errores podrían haberse categorizado como sintácticos si se considerara que -miento se usaba como sufijo adverbial. Con ambas interpretaciones se confirman las confusiones en el uso de estos dos sufijos debido a su parecido formal.

Los errores sintácticos de posición son solo dos. Uno de ellos es el siguiente: me gusta mucho su manera de pensar, su *tanquilidad, su *educacion, tratamiento *mi problemas en la casa (árabe, A1-A2), donde vemos que nos falta un determinante y también una preposición para el sintagma. 


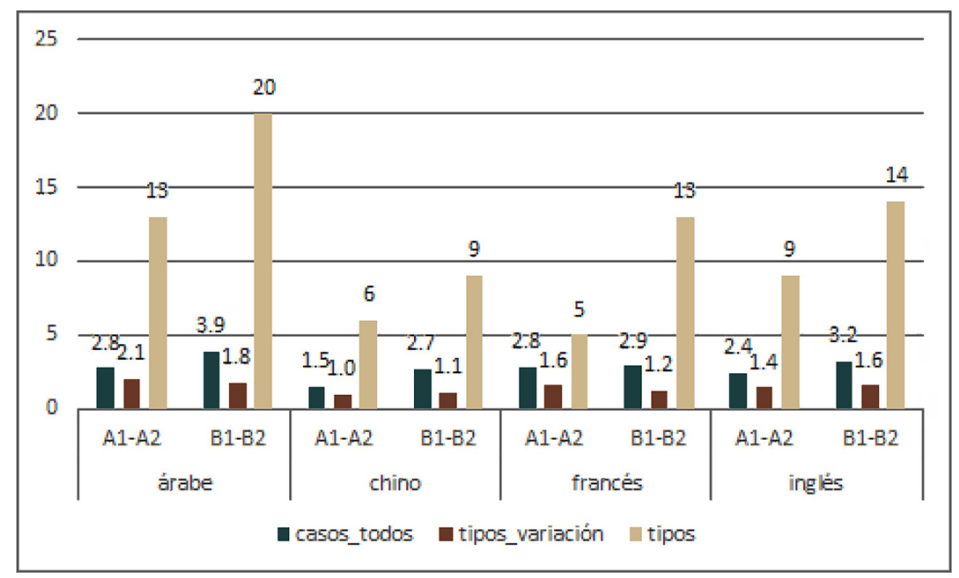

Figura 2. Medias de casos y variación acompañadas de la suma de tipos de palabras con -miento.

Los errores semánticos tratan aquellos casos donde sería difícil adivinar el significado de la palabra para un hispanohablante monolingüe; ej., *tournamento $\mathrm{y}^{*}$ contenimientos. Tan solo se dan estos dos casos. Estos errores semánticos se han clasificado también como errores en la base ya que eran dos bases inexistentes en español y podrían presentar dificultades para que hispanohablantes monolingües los comprendieran.

\begin{tabular}{|c|c|c|c|c|c|c|c|c|c|c|c|c|c|c|c|c|}
\hline \multirow[b]{4}{*}{ error } & \multicolumn{16}{|c|}{$\begin{array}{l}\text { ABLA 7. MEDIAS Y DESVIACIONES ESTÁNDARES DE ERRORES FORMALES } \\
\text { (ORTOGRÁFICOS, MORFOLÓGICOS Y SINTÁCTICOS) Y SEMÁNTICOS } \\
\text { CON PALABRAS CON -MIENTO }\end{array}$} \\
\hline & \multicolumn{4}{|c|}{ ÁrABE } & \multicolumn{4}{|c|}{ Сhino } & \multicolumn{4}{|c|}{ FRANCÉS } & \multicolumn{4}{|c|}{ INGLÉS } \\
\hline & \multicolumn{2}{|c|}{ A1-A2 } & \multicolumn{2}{|c|}{ B1-B2 } & \multicolumn{2}{|c|}{ A1-A2 } & \multicolumn{2}{|c|}{ B1-B2 } & \multicolumn{2}{|c|}{$\mathrm{A} 1-\mathrm{A} 2$} & \multicolumn{2}{|c|}{ B1-B2 } & \multicolumn{2}{|c|}{ A1-A2 } & \multicolumn{2}{|c|}{ B1-B2 } \\
\hline & M & $\mathrm{DE}$ & M & $\mathrm{DE}$ & M & $\mathrm{DE}$ & $\mathrm{M}$ & $\mathrm{DE}$ & $\mathrm{M}$ & $\mathrm{DE}$ & $\mathrm{M}$ & $\mathrm{DE}$ & $\mathrm{M}$ & $\mathrm{DE}$ & M & $\mathrm{DE}$ \\
\hline ortográfico & .77 & .92 & .35 & .67 & .17 & .41 & .11 & .33 & .20 & .45 & .08 & .28 & .00 & .00 & .14 & .36 \\
\hline vocal & .54 & .78 & .25 & .55 & .00 & .00 & .00 & .00 & .20 & .45 & .00 & .00 & .33 & .50 & .14 & .36 \\
\hline base & .08 & .23 & .00 & .00 & .00 & .00 & .00 & .00 & .20 & .45 & .08 & .28 & .22 & .44 & .07 & .27 \\
\hline sint. cat. & .00 & .00 & .00 & .00 & .00 & .00 & .11 & .33 & .00 & .00 & .00 & .00 & .00 & .00 & .07 & .27 \\
\hline sint. pos. & .15 & .38 & .00 & .00 & .00 & .00 & .00 & .00 & .00 & .00 & .08 & .28 & .00 & .00 & .00 & .00 \\
\hline sem. & .00 & .00 & .00 & .00 & .00 & .00 & .00 & .00 & .00 & .00 & .00 & .00 & .11 & .33 & .00 & .00 \\
\hline
\end{tabular}

sint.=sintáctico; cat.=categoría; pos.=posición; sem.=semántico

En principio, apenas se aprecian errores semánticos o sintácticos. Los errores ortográficos y morfológicos, aunque tampoco excesivos, son mucho más frecuentes que los errores semánticos y sintácticos. En especial, los hablantes de árabe e inglés parecen tener más dificultades o, dicho de otra manera, manipulan más las 
dos partes de las palabras con -miento y de ahí se genera lo que percibimos como errores. Los hablantes de chino se limitan a errores ortográficos.

\section{4. - Miento: Tipos y Similitudes Entre Lenguas}

Las tablas 8 y 9 representan los tipos con -miento en más de tres lenguas en nivel elemental e intermedio respectivamente. El número de palabras aumenta en total al igual que las lenguas compartidas. En negrita se marcan las palabras en común en todos los niveles y en más de tres lenguas.

\begin{tabular}{|c|c|c|c|}
\hline \multicolumn{4}{|c|}{$\begin{array}{l}\text { TABLA 8. PALABRAS EN A1-A2 CON -MIENTO EN MÁS DE TRES LENGUAS } \\
\text { CON MÁS DE UNA VARIACIÓN DE SU FORMA EN AL MENOS UNA DE LAS LENGUAS }\end{array}$} \\
\hline Nivel & Palabra & VARIACIÓN EN CADA LENGUA & LENGUAS (3 O MÁs) \\
\hline \multirow{5}{*}{$\begin{array}{l}\text { A1-A2 } \\
\text { (24 palabras únicas; } 7 \text { pala- } \\
\text { bras en dos lenguas o más; y } \\
5 \text { palabras en tres lenguas o } \\
\text { más con más variación que } \\
\text { una forma) }\end{array}$} & alojamiento & 1 a 2 & 3 \\
\hline & apartamento & 2 a 4 & 3 \\
\hline & comportamiento & 1 a 3 & 3 \\
\hline & departamento & 1 a 4 & 3 \\
\hline & nacimiento & 1 a 2 & 3 \\
\hline
\end{tabular}

\begin{tabular}{|c|c|c|c|c|}
\hline \multicolumn{5}{|c|}{$\begin{array}{l}\text { TABLA 9. PALABRAS EN B1-B2 CON -MIENTO EN MÁS DE TRES LENGUAS } \\
\text { CON MÁS DE UNA VARIACIÓN DE SU FORMA EN AL MENOS UNA DE LAS LENGUAS }\end{array}$} \\
\hline Nivel & Palabra & $\begin{array}{l}\text { VARIACIÓN EN } \\
\text { CADA LENGUA }\end{array}$ & $\begin{array}{l}\text { LENGUAS } \\
\text { (3 o MÁs) }\end{array}$ & $\begin{array}{l}\text { EJEMPLOS CON ERRORES } \\
\text { FORMALES }\end{array}$ \\
\hline \multirow{5}{*}{$\begin{array}{l}\text { B1-B2 } \\
\text { (hay } 39 \text { tipos en total; } \\
\mathbf{1 2} \text { aparecen en dos o más } \\
\text { lenguas, y } \mathbf{6} \text { aparecen en } \\
\text { tres o más lenguas y } 5 \\
\text { tienen más de una forma } \\
\text { para cada tipo) }\end{array}$} & alojamiento & 1 a 4 & 4 & alojemiento \\
\hline & apartamento & 1 a 2 & 3 & apartamenta \\
\hline & conocimiento & 2 a 4 & 4 & $\begin{array}{l}\text { conocimiemtos } \\
\text { conocimentos }\end{array}$ \\
\hline & departamento & 1 a 5 & 4 & $\begin{array}{l}\text { departamiento } \\
\text { departementio } \\
\text { departemento } \\
\text { departmento }\end{array}$ \\
\hline & sentimiento & 1 a 4 & 3 & sentiemento \\
\hline
\end{tabular}

Al comparar las tablas 5 y 6 de -mente con las de -miento (tabla 8 y 9) se aprecia cómo -mente presenta más tipos y manipulaciones. No obstante, las cuatro tablas presentan problemas ortográficos al igual que morfológicos, especialmente debido a la vocal temática, en las palabras derivadas examinadas. 


\section{DISCUSIÓN}

Ahora podemos revisar de nuevo las preguntas de investigación. Se contestará a la vez a las dos secciones de la primera pregunta ya que están estrechamente relacionadas, Conforme avanza el nivel de competencia de español en hablantes de ELE, ¿cómo se desarrolla el conocimiento 1 . de la forma (ortográfica, morfológica y sintáctica) y 2. del significado de palabras con los sufijos -mente y -miento? Como en la exposición de resultados, nos centraremos cada vez en un sufijo.

Según las características con -mente en relación con los sufijos de las L1 analizadas (ver tabla 1), la falta de similitud gráfica con -mente en árabe e inglés no impide que los aprendices de estas lenguas lo reconozcan y manipulen. Al parecer, el poder equipararlo semánticamente con un sufijo en la L1 es más importante. Como se había hipotetizado, los hablantes de chino L1 son los que menos descomponen la palabra en sus elementos. No descartamos que esto se deba a influencias externas a la L1 como el tipo de enseñanza que reciben, que les hace estar más dispuestos a manipular la L2. Por ejemplo, se ha visto que estudiantes con coreano como L1 y árabe como L1 aprenden vocabulario de manera diferente (Juff y Friedline, 2014). No podemos contestar esta pregunta con la información recogida en este estudio, pero es un aspecto para explorar en el futuro comparando las influencias socioculturales de los aprendices con árabe como L1 y chino como L1.

En general vemos que la variación ortográfica con-mente disminuye en todas las lenguas al aumentar el nivel de competencia (figura 1), pero sigue habiendo variación en los niveles B1-B2. Todos los errores formales también disminuyen al aumentar el nivel de competencia, pero no desaparecen (ver tabla 4). Los errores sintácticos y semánticos son mucho menores que los formales a pesar de que al haberlos analizado por casos teníamos más ejemplos que con los ortográficos y morfológicos que se han analizado por tipos de palabras.

Con -miento, hay menos ejemplos, tanto de casos como de tipos, de palabras derivadas. La media de errores formales es mayor que la de los semánticos. En errores ortográficos, se ven unos datos similares a -mente mientras que para errores morfológicos aparecen menos que con -mente. Las medias de errores semánticos y sintácticos son similares entre los dos sufijos: muy bajas. En general, se ve una disminución de errores con -miento al aumentar la competencia de los hablantes (ver tabla 7). No obstante, los errores sintácticos, aunque en un número muy limitado, aparecen más en niveles intermedios. Al igual que con -mente, los hablantes de chino cometen menos errores, lo que sugiere que estos sufijos no se manipulan como elementos independientes al escribir la palabra.

En el caso de -miento nos encontramos que la alomorfía -miento y -mento causa dificultades a los aprendices (ej.. ${ }^{*}$ conocimento en vez de conocimiento). A pesar de que la forma -miento es la más productiva de las dos en la actualidad (Lang, 2009), esto no evita la alternancia de formas. Por ejemplo, del latín complementum tenemos complemento, complimiento y cumplimiento (Cervera Rodríguez, 2012). Por tanto, no podemos esperar que los aprendices distingan qué forma usar a pesar de que hayan sido capaces de reconocer y producir el sufijo. De hecho, el confundir los alomorfos sugiere una consciencia sobre la relación de los dos como alomorfos. 
En cierta manera, no podemos separar totalmente el conocimiento de la ortografía de la morfología ya que el producir y reconocer la forma del sufijo entra dentro de la conciencia morfológica del estudiante (ver Fejzo, 2016; Kuo y Anderson, 2006; Llombart-Huesca, 2018). No obstante, de la sistematicidad de los errores con la vocal antes del sufijo, tanto por niveles como por lenguas (ej., *actualamente), deducimos que los aprendices están negociando la formación de la palabra y no es solo una mera confusión ortográfica.

En resumen, el significado de las palabras derivadas tiende a aprenderse en relación con la palabra total y no con la suma de sus partes. Esta situación causa que haya más errores formales que semánticos en el momento de la producción escrita que aquí se analiza. En los ejemplos revisados, los aprendices parecen descomponer la palabra y de ahí que se produzcan errores ortográficos y morfológicos con relativa frecuencia. Es decir, similar a Jiang (2000), podemos establecer tres pasos del aprendizaje: 1. aprendizaje semántico de la palabra, donde se equipara a una palabra en la L1; 2. aprendizaje formal de los elementos de la palabra (base y sufijo), donde se empiezan a desarrollar conexiones en la L2; y 3. aprendizaje del significado del sufijo, donde esperamos nuevas conexiones en la L2. Estas etapas podrían darse simultáneamente según las palabras y el aprendiz. No obstante, los datos del corpus no ofrecen suficiente información sobre la tercera etapa. Análisis posteriores con datos empíricos de participantes con distintas L1 ayudarían a contestar esta pregunta.

Para la segunda pregunta, ¿Qué sufijo, -mente o-miento, presenta más dificultades para los estudiantes? Es decir, ¿dónde deberian insistir más los profesores? ¿Qué palabras pueden usar como ejemplos prototípicos?, podemos ahora concluir que, en términos de producción, los dos sufijos pueden presentar problemas con su ortografía. No obstante, por su reconocimiento y productividad el sufijo -mente presenta más errores formales y semánticos. En el caso de los errores semánticos estos son específicos de cada palabra y, por tanto, parece más difícil sistematizarlos. Por ejemplo, basado en los ejemplos, recomendamos a los profesores revisar el significado de la palabra últimamente.

Las tablas 5-6 (-mente) y 8-9 (-miento) recogen los ejemplos formales que permitirán a los profesores mayor reflexión en el aula. Este tipo de tareas se recogerían dentro del tiempo de la clase dedicado a la forma y las estructuras de la lengua (ver Nation, 2007). El indicarles a los estudiantes que los errores están basados en composiciones de otros estudiantes siempre resulta motivante, pues los aprendices reconocerán que estas dificultades las podrían enfrentar ellos mismos. A continuación, se dan dos modelos para actividades de reflexión lingüística con ejemplos obtenidos en este análisis.

\section{Modelo 1:}

De las siguientes palabras con -mente solo una es correcta. Reflexiona con tus compañeros. Explicad por qué funciona el ejemplo elegido y por qué son incorrectas las otras opciones.

facialmente facilamente facilemente facilmente fácilmente 
Modelo 2:

Las palabras con -mente no se usan correctamente en algunas de las siguientes oraciones. Reflexiona con tus compañeros y proponed sugerencias para mejorar las oraciones.

a. Saludos cordialmente. [En una carta]

b. Necesitamos una (habitación) doble con dos baños independientes. Si possiblement, debería tener un minibar y una vista al mar.

c. Ven a Nueva York. Es una ciudad muy interesante, muy rápidamente.

[ejemplos levemente revisados de CAES para evitar los errores que no se refieren a-mente]

\section{CONCLUSIONES}

Este estudio ha examinado el proceso de adquisición de los sufijos -mente y -miento en aprendices de español como L2. Para este fin, se han estudiado hablantes de L1 árabe, chino, francés e inglés en el corpus CAES de nivel principiante e intermedio.

Los resultados demuestran que, en el aprendizaje de sufijos, más que su significado, lo que conlleva más dificultades a la hora de usarlos es su forma. Por tanto, se recomienda trabajar la forma en la clase de los sufijos (ortográfica, morfológica y sintáctica) para todos los aprendices de español L2. Especialmente, se recomienda enfatizar la estructura de las palabras derivadas para los estudiantes cuyas lenguas no utilizan una estructura similar al espańol, ya que su L1 puede limitar su habilidad para descomponer palabras derivadas como hemos visto con hablantes de chino L1. Entendemos que en el aula hay un tiempo limitado, pero como las actividades modelos propuestas son muy breves, se pueden incluir dentro de la instrucción habitual de vocabulario. Debido a la frecuencia de palabras derivadas con -mente y -miento, el profesor encontrará ocasiones para reflexionar sobre la forma sin forzar el tema.

Este estudio cuenta con ciertas limitaciones especialmente debidas al tamaño del corpus CAES, a que no presenta el mismo número de casos por lengua y nivel y a que no se han tenido en cuenta las variaciones por hablante. También sería importante incorporar datos empíricos sobre el conocimiento de los sufijos -mente y -miento para contrastar la información. Por ejemplo, la aparente falta de manipulaciones morfológicas en hablantes chinos podría deberse a que estos utilizan una estrategia de memorización de las palabras como hemos dicho, pero no se puede afirmar que no hayan adquirido los sufijos. Los datos de corpus reflejan en su totalidad el uso de los sufijos y de ahí inferimos el proceso de adquisición. 


\section{BIBLIOGRAFÍA}

Baralo, Marta (1996): «Algunos aspectos de la adquisición de la morfología léxica del ELE», en Tendencias actuales en la enseñanza del español como lengua extranjera I: Actas del quinto Congreso Internacional de ASELE: Santander, 29, 30 de septiembre y 1 de octubre de 1994. Asociación para la Enseñanza del Español como Lengua Extranjera, 143-150.

Callies, Marcus (2016): «Towards a process-oriented approach to comparing EFL and ESL varieties", International Journal of Learner Corpus Research 2(2): 229-251.

Cervera Rodríguez, Ángel (2012): «Los sufijos nominalizadores -ción, -sión, -miento/-mento en el español actual», Revista Cálamo FASPE 60: 47-52.

Clahsen, Harald y Claudia Felser (2017): «Some notes on the shallow structure hypothesis», Studies in Second Language Acquisition, 40(3): 693-706.

Díaz Hormigo, María Tadea (2011): «Formación de palabras y ELE: una propuesta didáctica para la enseñanza de la formación de verbos por prefijación en español a discentes italianos», redELE: Revista Electrónica de Didáctica ELE 22: 25-46.

FeJzo, Anila (2016): «The contribution of morphological awareness to the spelling of morphemes and morphologically complex words in French", Reading and Writing, 29: 207-228.

Fernández, Claudia (1998): «La creación léxica en la interlengua de español», en Lengua y cultura en la enseñanza del español a extranjeros: Actas del VII Congreso de ASELE. Ediciones de la Universidad de Castilla-La Mancha, 213-220.

JiANG, Nan (2000): «Lexical representation and development in a second language», Applied linguistics 21(1): 47-77.

Juffs, Alan y Benjamin E. Friedline (2014): «Sociocultural influences on the use of a web-based tool for learning English vocabulary", System, 42: 48-59.

Kuo, Li-jen and Richard C. ANDERson (2006): «Morphological awareness and learning to read: A cross-language perspective», Educational Psychologist, 41(3): 161-180.

LANG, Mervin (2009): Formación de palabras en español. Morfología derivativa productiva en el léxico moderno, Madrid: Cátedra.

Llombart-Huesca, Amalia (2018): «Understanding the spelling errores of Spanish heritage language learners», Hispania, 101(2): 211-223.

Lozano, Cristóbal y Ana DíAz-NEgrillo (2019): «Using learner corpus methods in L2 acquisition research. The morpheme order studies revisited with Interlanguage Annotation", Revista Española de Lingüistica Aplicada/Spanish Journal of Applied Linguistics 32(1): 82-124.

Lüdeling, Anke, Hagen Hirschmann y Anna Shadrova (2017): «Linguistic models, acquisition theories, and learner corpora: Morphological productivity in SLA research exemplified by complex verbs in German", Language Learning 67(S1): 96-129.

Marcos Miguel, Nausica (2016): «Voluntad y viabilidad en la enseñanza de morfología léxica en el aula de español como L2», en Santiago Alcoba, Cristina Buenafuente y Gloria Clavería (eds.), Los lindes de la morfología 37, Pontevedra: Universidade da Coruña, Servizo de Publicacións, 145-158.

Marcos Miguel, Nausica (2017): «Instruction in derivational morphology in the Spanish L2 Classroom: What do teachers believe and do?», Konin Language Studies 5(1): 37-60.

Marcos Miguel, Nausica (2018): "Analyzing the relationship and development of proficiency, derivational knowledge, and vocabulary size in Spanish L2 learners", Revista Española de Lingüistica Aplicada / Spanish Journal of Applied Linguistics 31(1): 224-256. 
Martínez VÁzQUeZ, Julián (2016): «Importancia de trabajar la competencia morfológica en las clases de español: la derivación», Gramma 26(55): 127-134.

Meara, Paul e Inmaculada Miralpeix (2016): Tools for researching vocabulary, Bristol: Multilingual Matters.

Montero Curiel, Marisa (2009): «La enseñanza del sistema sufijal español a estudiantes extranjeros como herencia de la gramática tradicional y de los manuales escolares», en David SerranoDolader, María Antonia Martín Zorraquino y José Francisco Val Álvaro (eds.), Morfología y español como lengua extranjera (E/LE), Zaragoza: Prensas Universitarias de Zaragoza, 91-116.

Morin, Regina (2003): «Derivational morphological analysis as a strategy for vocabulary acquisition in Spanish", The Modern Language Journal 87(2): 200-221.

Morin, Regina (2006): «Building depth of Spanish L2 vocabulary by building and using word families», Hispania 96(3): 170-182.

Murakami, Akira y Theodora Alexopoulou (2016): «L1 influence on the acquisition order of English gramatical morphemes: A learner corpus studies», Studies in Second Language Acquisition, 38(3): 365-401.

Nation, Paul (2007): «The four strands», International Journal of Innovation in Language Learning and Teaching, 1(1): 2-13.

Robles García, Pablo y Claudia Helena Sánchez Gutiérrez (2016): «La morfología derivativa en los manuales de español elemental estadounidenses: Un estudio exploratorio», RAEL: Revista Electrónica de Lingüistica Aplicada 15(1): 70-86.

Rojo, Guillermo e Ignacio M. Palacios Martínez (2016): «Learner Spanish on computer», en Margarita Alonso-Ramos (ed.), Spanish Learner Corpus Research: Current trends and future perspectives, Amsterdam: John Benjamins, 55-88.

SÁnchez Gutiérrez, Claudia Helena (2013): Priming morfológico y conciencia morfológica: una investigación con estudiantes norteamericanos de ELE, tesis doctoral, Universidad de Salamanca.

Sánchez Gutiérrez, Claudia Helena, Nausica Marcos Miguel y Pablo Robles García (2020): "What derivational suffixes should we teach in Spanish as a Second Language courses?», en Alfonso Morales-Front, Michael J. Ferreira, Ronald P. Leow y Cristina Sanz (eds.), Hispanic Linguistics: Current Issues and New Directions. Issues in Hispanic Linguistics, 26. Amsterdam: John Benjamins: 75-94.

SÁnchez Gutiérrez, Claudia Helena y Natividad Hernández Muñoz (2018): «Development of derivational morphological awareness in anglophone learners of Spanish: A relational knowledge study", Foreign Language Annals 51(2): 369-388.

Serrano-Dolader, David (2019): Formación de palabras y enseñanza del español LE/L2, Oxon: Routledge.

Serrano-Dolader, David (s.f.): La formación de palabras en la clase de ELE. Antología de Textos de Didáctica del Español: Centro Virtual del Instituto Cervantes. URL: https://cvc.cervantes. es/ensenanza/biblioteca_ele/antologia_didactica/gramatica/default.htm.

TYLER, Andrea y William NAGY (1989): «The acquisition of English derivational morphology», Journal of memory and language 28(6): 649-667.

WhitLey, Stanley (2004): «Lexical errors and the acquisition of derivational morphology in Spanish», Hispania 87(1): 163-172.

Zyzik, Eve y Clara Azevedo (2009): «Word class distinctions in second language acquisition: An experimental study of L2 Spanish», Studies in Second Language Acquisition 31(1): 1-29. 
